\title{
Prevalence of vitamin B12 deficiency in type 2 diabetes mellitus patients on metformin therapy in a tertiary care hospital
}

\author{
Gomathi A.*, Keerthiga K.
}

Department of Pharmacology, Government Vellore Medical College, Vellore, Tamil Nadu, India

\author{
Received: 30 October 2020 \\ Revised: 12 November 2020 \\ Accepted: 13 November 2020 \\ *Correspondence: \\ Dr. Gomathi A, \\ Email: goms1180@gmail.com
}

Copyright: (c) the author(s), publisher and licensee Medip Academy. This is an open-access article distributed under the terms of the Creative Commons Attribution Non-Commercial License, which permits unrestricted non-commercial use, distribution, and reproduction in any medium, provided the original work is properly cited.

\begin{abstract}
Background: The objective of the study was to estimate the prevalence of vitamin B12 deficiency and presence of peripheral neuropathy in type 2 diabetes patients on metformin treatment.

Methods: This cross-sectional study was carried out in Department of Biochemistry and Medicine in a tertiary care teaching hospital in south India. Type 2 diabetes mellitus patients diagnosed based on World Health Organization (WHO) criteria treated with metformin for more than 6 months were included in the study. B12 levels were estimated and DNS scoring, vibration perception test and $10 \mathrm{gm}$ Semmes-Weinstein monofilament test done.

Results: A total of 55 participants were identified. Most of them were on $1500 \mathrm{mg}$ of metformin once a day in divided dose. In this study maximum number of participants are in the age group of 50-70 years. Peripheral neuropathy is seen in $43.9 \%$ of persons with normal B12 levels and $28.5 \%$ of persons with low B12 levels. A Chi square test showed a chi square statistic value of 1.8221 with a $\mathrm{p}$ value of 0.312 which is not significant. Univariate analysis showed vitamin B12 deficient patients are of significantly higher age (63 years versus 57 years $\mathrm{p}=0.01$ ) and with higher duration of treatment ( 9 years versus 6 years $\mathrm{p}=0.01$ ).

Conclusions: Prolonged metformin therapy is associated with deficiency of vitamin B12. The prevalence of B12 deficiency among diabetic patients on metformin was found to be $25.45 \%$. Routine B12 estimation and correction of low B12 levels will be beneficial to these patients. Earlier initiation of B12 in patients on metformin may prevent the onset of neurological damage.
\end{abstract}

Keywords: Metformin, B12 deficiency, Diabetes mellitus

\section{INTRODUCTION}

Metformin is one of the most commonly used oral anti diabetic drug in type 2 diabetes. Metformin is the firstchoice drug after initial diagnosis of type 2 diabetes. ${ }^{1}$ Metformin is well tolerated in most of the patients and common side effects which include mild gastrointestinal symptoms like anorexia, nausea, vomiting, diarrhoea etc. are mostly transient. ${ }^{2}$ However long term treatment results in decreased vitamin B12 absorption which may cause in B12 deficiency.
A randomised placebo-controlled trial by de Jager et al looking at long term treatment with metformin in patients with type 2 diabetes showed a prevalence of $9.9 \%$ in 196 patients. ${ }^{3}$ Reinstatler et al found a prevalence of $5.8 \%$ in 598 patients. ${ }^{4}$ A cross-sectional cohort study done by Ermann et al had prevalence of $8 \%$ in 53 patients. ${ }^{5}$ Prevalence of $14.1 \%$ in 164 patients was seen in the study by de Groot-Kamphuis et al. ${ }^{6}$ Romero et al, confirmed a prevalence of $8.6 \%$ in 81 patients. $^{7}$ In 2014 , Beulens et al estimated a prevalence of $28.1 \%$ in 550 patients of Netherlands. ${ }^{8}$ Ahmed et al found a prevalence of $28.1 \%$ in 121 patients of south African origin. ${ }^{9}$ 
Long term undetected B12 deficiency can cause neurological damage and megaloblastic anaemia. ${ }^{10}$ Hence it is important to identify B12 deficiency in metformin treated diabetic patient for prevention of irreversible neurological damage. ${ }^{11}$ So the study was planned to estimate the prevalence of vitamin B12 deficiency and presence of peripheral neuropathy in type 2 diabetes patients on metformin treatment.

\section{Objectives}

The objectives of the study was to estimate the prevalence of vitamin B12 deficiency and presence of peripheral neuropathy in type 2 diabetes patients on metformin treatment.

\section{METHODS}

This cross-sectional study was carried out in Department of Biochemistry and the department of Medicine (Outpatient Department) in a Tertiary care teaching hospital in south India.

This study was approved by the Institutional ethics committee (IEC) of Government Vellore Medical College.

\section{Study population}

Type 2 diabetes mellitus patients diagnosed based on World Health Organization (WHO) criteria treated with metformin for more than 6 months.

\section{Duration of study}

The duration of the study was 2 months, June to July 2019.

\section{Sample size}

Convenient sample was 55 patients.

Adults who attend diabetes outpatient clinic in the department of Medicine in our tertiary care institution, were randomly selected and explained about the procedure. Among them, those who fulfilled the inclusion criteria and willing to participate in this study were recruited after obtaining written informed consent. A total of 100 patients who attended the outpatient clinic were screened and 55 patients who fulfilled the criteria were enrolled for the study.

\section{Inclusion criteria}

Age above 18 years, gender: both male and female, patients diagnosed as type 2 diabetes mellitus based on WHO criteria on metformin treatment for more than 6 months and patients willing to give informed consent were included.

\section{Exclusion criteria}

Patients not willing to give informed consent, patients with gastrectomy, small bowel resection, liver disease, chronic kidney disease and thyroid disease, vegetarians and patient on $\mathrm{H} 2$ receptor blocker and vitamin B supplementation were excluded.

The demographic data was collected, followed by clinical examination and lab tests as given below.

\section{Demographic data}

The following demographic data was collected- age, gender, dietary habits, smoker/non-smoker, time since diagnosis of diabetes and known comorbidities related to metabolic syndrome.

\section{Clinical examination}

Clinical examination included test for sensations, and vibration perception in the feet.

\section{Sensation status using $10 \mathrm{gm}$ Semmes-Weinstein monofilament}

\section{Clinical testing}

Sensation status was determined by applying a $10 \mathrm{gm}$ Semmes-Weinstein monofilament perpendicular to the skin, until the filament bends, for 1 second, to 4 points on the plantar surface of each foot of blindfolded patientsgreat toe, 1st Metatarsal head, 5th metatarsal head and heel pad.

Patients were explained the procedure once prior to proper testing and were expected to indicate when they perceive the application of the monofilament. Those who failed to sense the monofilament at one or more locations were tested once again before being classified as insensate.

\section{Interpretation}

Consistent classification as being insensate in at least one of the four tested points was deemed as positive screening test for presence of peripheral neuropathy.

Vibration perception using calibrated $128 \mathrm{~Hz}$ tuning fork

\section{Clinical testing}

Vibration perception was determined by applying the base of a vibrating $128 \mathrm{~Hz}$ calibrated tuning fork to the dorsal aspect of the interphalangeal joint of the great toe of each foot of the blindfolded patients. Patients were explained the procedure once prior to proper testing and were be expected to indicate when they perceive the application of vibrations, and also indicate when they feel the vibrations 
have stopped. Those who failed to sense the monofilament at one or more locations were tested once again.

\section{Interpretation}

Failure to sense the vibrations, or failure to indicate the correct timing of dampening of the vibrations in either of the feet as judged by the examiner was deemed as positive screening test of presence of impaired vibratory perception sense.

\section{Semi-structured neuropathy pain questionnaire}

Patients were asked to complete semi-structured questionnaire to know their subjective experience of neuropathy related symptoms such as numbness, tingling and altered skin characteristics.

\section{Vitamin B12 estimation}

Venous peripheral blood sample $2 \mathrm{ml}$ was drawn from all participants for estimation of serum B12 by using chemiluminescent enzyme immunoassay method. Serum B12 $<180$ pg/dl was considered as deficiency.

\section{Statistical analysis}

Clinical characteristics and parameters were summarized as the mean \pm standard deviation (SD), or numbers (percentage).

Pearson's chi-square tests was used to test the differences in the proportion of categorical variables.

Pearson correlation analyses was performed to examine the linear relationship between serum vitamin B12 and metformin use. ' $\mathrm{R}$ ' statistical software was used for analysis.

\section{RESULTS}

A total of 55 participants were identified. All of them were on $1500 \mathrm{mg}$ of metformin daily in divided doses. In this study maximum number of participants are in the age group of 50-70 years. The participant's demographic and clinical details are as follows: mean age in years $=56.7( \pm 9.5)$, duration of T2 DM (years) $=7.3 \quad( \pm 2.3)$, duration of metformin use (years) $=6.9 \quad( \pm 3.2)$, mean serum B12 level $=330.9( \pm 156.6)$, persons with B12 deficiency $=14$ $(25.45 \%)$ and persons with peripheral neuropathy $=22$ (40\%).

Table 1: The relationship between peripheral neuropathy and B12 levels.

\begin{tabular}{|lll|} 
& $\begin{array}{l}\text { Normal } \\
\text { B12 level }\end{array}$ & Low B12 level \\
\hline Neuropathy present & 18 & 4 \\
\hline Neuropathy absent & 23 & 10 \\
\hline
\end{tabular}

The relationship between peripheral neuropathy and B12 levels is described in table 1 .

Peripheral neuropathy is seen in $43.9 \%$ of persons with normal B12 levels and $28.5 \%$ of persons with low B12 levels. A Chi square test showed a chi square statistic value of 1.8221 with a $\mathrm{p}$ value of 0.312 which is not significant.

Univariate analysis showed vitamin B12 deficient patients are of significantly higher age (63 years versus 47 years $\mathrm{p}=0.01$ ) and with higher duration of treatment (9 years versus 6 years $\mathrm{p}=0.01$ ).

\section{DISCUSSION}

Vitamin B12 deficiency associated with metformin use is thought to occur due to vitamin B12 malabsorption. Initial theories included alteration of bile acid metabolism, small intestinal bacterial overgrowth, or effects on intrinsic factor secretion, but a more currently accepted explanation is the interference by metformin on calcium-dependent membrane action responsible for vitamin B12-intrinsic factor absorption in the terminal ileum. ${ }^{12-14}$

The present study has found that the prevalence of B12 deficiency among diabetic patients on metformin is $25.45 \%$. The B12 deficiency is more in higher age of the patient $(\mathrm{p}=0.01)$ and greater duration of treatment with metformin $(\mathrm{p}=0.01)$.

A cross-sectional cohort study done by Ermann, et al had prevalence of $8 \%$ in 53 patients. $^{7}$ Prevalence of $14.1 \%$ in 164 patients was seen in the study by de Groot-Kamphuis et al. ${ }^{8}$ Romero, confirmed a prevalence of $8.6 \%$ in 81 patients. ${ }^{9}$ In 2014, Beulens et al estimated a prevalence of $28.1 \%$ in 550 patients of Netherlands. ${ }^{10}$ While various studies have reported a prevalence of B12 deficiency between $8-30 \%$, a prevalence of $25 \%$ is found in this study. The prevalence of B12 deficiency is significantly higher with increasing age and higher duration of treatment.

However, association between B12 deficiency and peripheral neuropathy is not significant as in the present study. Peripheral neuropathy is seen in $43.9 \%$ of persons with normal B12 levels and $28.5 \%$ of persons with low B12 levels. A Chi square test showed a chi square statistic value of 1.8221 with a $\mathrm{p}$ value of 0.312 which is not significant. Peripheral neuropathy was seen in patients with normal B12 levels also. This might be due to the duration of the diabetes mellitus in these patients and also due to late diagnosis of diabetes. Some patients with B 12 deficiency did not have peripheral neuropathy as they would have developed B12 deficiency recently, or the peripheral neuropathy has not yet set in. Vitamin B12 deficiency due to metformin use maybe not related to neuropathy if it is a simple plasma deficiency. ${ }^{15}$ Although it is difficult to clinically distinguish neuropathy caused by vitamin B12 deficiency from diabetic neuropathy, neuropathy should be closely monitored as early diagnosis and treatment improve prognosis. 
So far no standard guidelines are available for routine B12 deficiency screening in patients on metformin. Further indepth studies may be required to recommend routine screening.

\section{Limitations}

The study was done in a tertiary care setting where more complicated patients are expected. There was not much variation in the metformin dosage between the patients. A larger study with more participants and measurement of more markers like homocysteine and other associated factors may yield a better result.

\section{CONCLUSION}

We can conclude that prolonged metformin therapy is associated with deficiency of vitamin B12.The prevalence of B12 deficiency among diabetic patients on metformin was found to be $25.45 \%$ in our study. Routine B 12 estimation and correction of low B 12 levels will be beneficial to the patients on long term metformin therapy. Earlier initiation of B12 in patients on metformin may prevent the onset of neurological damage.

\section{ACKNOWLEDGEMENTS}

Authors would like to thank Department of Biochemistry, GVMCH, Vellore.

\section{Funding: No funding sources}

Conflict of interest: None declared

Ethical approval: The study was approved by the Institutional Ethics Committee

\section{REFERENCES}

1. Katzung, Trevor, Masters. Basic and Clinical Pharmacology. 12 ${ }^{\text {th }}$ edition. Mc-Graw Hill. 2010.

2. American Diabetes Association. Standards of medical care in diabetes: 2013. Diabetes Care. 2013;36:S1166.

3. de Jager J, Kooy A, Lehert P, WulffeleMG, van der Kolk J, Bets D, et al. Long term treatment with metformin in patients with type 2 diabetes and risk of vitamin B- 12 deficiency: randomised placebo controlled trial. BMJ. 2010;340:c2181.

4. Reinstatler L, Qi YP, Williamson RS, Garn JV, Oakley Jr GP. Association of biochemical B12 deficiency with metformin therapy and vitamin B12 supplements: the National Health and Nutrition
Examination Survey, 1999-2006. Diabetes Care. 2012;35(2):327-33.

5. Hermann LS, Nilsson B, Wettre S. Vitamin B12 status of patients treated with metformin: a cross-sectional cohort study. Br J Diabetes Vasc Dis. 2004;4:401-4.

6. de Groot-Kamphuis DM, van Dijk PR, Groenier KH, Houweling ST, Bilo HJ, Kleefstra N. Vitamin B12 deficiency and the lack of its consequences in type 2 diabetes patients using metformin. Neth $\mathrm{J}$ Med. 2013;71(7):386-90.

7. Calvo Romero JM, Lozano JM. Vitamin B (12) in type 2 diabetic patients treated with metformin. Endocrinol Nutr. 2012;59(8):487-90.

8. Beulens JW, Hart HE, Kuijs R, Kooijman-Buiting AM, Rutten GE. Influence of duration and dose of metformin on cobalamin deficiency in type 2 diabetes patients using metformin. Acta Diabetol. 2014;52(1):47-53.

9. Ahmed et al. Vitamin B12 deficiency in metformin treated type-2 diabetes patients, prevalence and association with peripheral neuropathy BMC Pharmacol Toxicol. 2016;17:44.

10. Bell DS. Metformin-induced vitamin B12 deficiency presenting as a peripheral neuropathy. South Med J. 2010;103:265-7.

11. Pierce SA, Chung AH, Black KK. Evaluation of vitamin B12 monitoring in a veteran population on long-term, high-dose metformin therapy. Ann Pharmacother. 2012;46:1470-6.

12. Caspary WF, Zavada I, Reimold W, Deuticke U, Emrich D, Willms B. Alteration of bile acid metabolism and vitamin-B12-absorption in diabetics on biguanides. Diabetologia. 1977;13:187-93.

13. Berchtold P, Dahlqvist A, Gustafson A, Asp NG. Effects of a biguanide (metformin) on vitamin B 12 and folic acid absorption and intestinal enzyme activities. Scand J Gastroenterol. 1971;6:751-54.

14. Bauman WA, Shaw S, Jayatilleke E, Spungen AM, Herbert V. Increased intake of calcium reverses vitamin B12 malabsorption induced by metformin. Diabetes Care. 2000;23:1227-31.

15. Ahmed MA, Muntingh GL, Rheeder P. Perspectives on peripheral neuropathy as a consequence of metformin-induced vitamin B12 deficiency in T2DM. Int J Endocrinol. 2017;2017:245-53.

Cite this article as: Gomathi A, Keerthiga $\mathrm{K}$.

Prevalence of vitamin B12 deficiency in type 2 diabetes mellitus patients on metformin therapy in a tertiary care hospital. Int J Basic Clin Pharmacol 2020;9:1888-91. 